\title{
Note on essential fixed points of approximable multivalued mappings
}

\author{
Jan Andres ${ }^{1 *}$ and Lech Górniewicz ${ }^{2}$
}

${ }^{\text {*Correspondence: }}$

jan.andres@upol.cz

'Department of Mathematical

Analysis and Applications of

Mathematics, Faculty of Science,

Palacký University, 17. listopadu 12,

Olomouc, 771 46, Czech Republic

Full list of author information is

available at the end of the article

\begin{abstract}
A new definition of essential fixed points is introduced for a large class of multivalued maps. Two abstract existence theorems are presented for approximable maps on compact ANR-spaces in terms of a nontrivial fixed point index, or a nontrivial Lefschetz number and a zero topological dimension of the fixed point set. The second one is applied to the periodic dissipative Marchaud differential inclusions for obtaining the existence of a discretely essential subharmonic solution. Three simple illustrative examples are supplied.
\end{abstract}

MSC: Primary 55M20; 54C60; 55M15; secondary 54H25; 47H04; 47H10; 34A60; 34C25

Keywords: essential fixed points; fixed point index; Lefschetz number; absolute neighborhood retracts; approximable multivalued mappings; J-maps; Poincaré translation operators; dissipative differential inclusions; discretely essential periodic solutions

\section{Introduction}

In the present note, we will consider for the first time the notion of essential fixed points to multivalued maps as defined below. More concretely, we will present two abstract theorems about the existence of essential fixed points to a large class of approximable multivalued maps, on compact ANR-spaces, in terms of a nontrivial fixed point index, or a nontrivial Lefschetz number and a zero topological dimension of the fixed point set.

These two theorems can be regarded as a multivalued generalisation of their analogies in our recent paper [1] ( $c f$. also [2], Section 12), where single-valued maps were exclusively examined for the same goal. On the other hand, unlike in [1, 2], we do not consider here compact multivalued maps, or even multivalued maps with only a certain amount of compactness like compact absorbing contractions, on arbitrary ANR-spaces. This remains as a challenge for our future research.

In our approach, we again follow the seminal ideas of Fort, Jr. and O'Neil in their classical papers [3, 4] from the early 1950s. Hence, roughly speaking, the fixed point, say $x_{0}$, of a given multivalued approximable mapping is essential if any continuous single-valued map which is sufficiently 'near' admits a fixed point in the neighborhood of $x_{0}$. For a precise formulation, see Definition 5.1 below, and for some further results in this field, see the references in [1]. Let us note that this definition significantly differs from all the other definitions for multivalued maps (see e.g. [5, 6] and the references therein), because it effectively employs the approximability of given multivalued maps on their graphs by single-valued

(c) 2016 Andres and Górniewicz. This article is distributed under the terms of the Creative Commons Attribution 4.0 International License (http://creativecommons.org/licenses/by/4.0/), which permits unrestricted use, distribution, and reproduction in any medium, provided you give appropriate credit to the original author(s) and the source, provide a link to the Creative Commons license, and indicate if changes were made. 
maps. In this way, topological invariants like a fixed point index can easily be calculated just by means of these single-valued approximations. This profit naturally connects our approach with the classical theory developed by Fort, Jr. [3] and O'Neil [4].

There are a lot of important applications of the essential fixed point theory like those in economy and the theory of games (see again the references in [1]). In [1], we concentrated to essential multivalued fractals considered as fixed points of the induced (single-valued) Hutchinson-Barnsley operators in hyperspaces. Here, our application concerns periodic solutions of periodic dissipative (in the sense of Levinson) differential inclusions. From our theoretical results, we will deduce that if a periodic dissipative system of Marchaud inclusions possesses at most a finite number of subharmonic periodic solutions or, in particular, entirely bounded solutions, then it admits a discretely essential periodic solution.

As already pointed out in [1], the essentiality can be regarded as a sort of structural stability which has a lot to do with the shadowing property for chaotic dynamics. Thus, the main profit consists not only in an additional information as regards the localization of fixed points of 'near' single-valued approximations, but in a numerical reliability at all.

In order to demonstrate the power of the obtained results, three simple illustrative examples are supplied.

\section{Preliminaries}

Let $X=(X, d)$ be a metric space. Let us recall that $X$ is an absolute neighborhood retract (written $X \in A N R$ ) if there exist an open set $U$ in a normed space and two single-valued continuous maps $r: U \rightarrow X$ and $s: X \rightarrow U$ such that $r \circ s=\mathrm{id}_{X}$, where $\mathrm{id}_{X}$ stands for the identity on $X$.

If $U$ is an arbitrary convex set, then $X$ is called an absolute retract (written $X \in \mathrm{AR}$ ). Evidently: AR $\subset$ ANR.

A compact space is called an $R_{\delta}$-set if it is an intersection of a decreasing sequence of compact AR-spaces. For compact sets, in particular: convex $\subset \mathrm{AR} \subset R_{\delta}$.

Let $X=\left(X, d_{X}\right)$ and $Y=\left(Y, d_{Y}\right)$ be metric spaces. By multivalued mappings $\varphi: X \multimap Y$, we understand here those with nonempty, closed values, i.e. that $\varphi: X \rightarrow 2^{Y} \backslash\{\emptyset\}$ and a closed set $\varphi(x) \subset Y$ is assigned to every point $x \in X$. By a fixed point of $\varphi: X \multimap Y$, we mean the point $x_{0} \in X \cap Y$ such that $x_{0} \in \varphi\left(x_{0}\right)$.

A mapping $\varphi: X \multimap Y$ is said to be upper semicontinuous (written u.s.c.) if, for every open $U \subset Y$, the set $\varphi^{-1}(U):=\{x \in X ; \varphi(x) \subset U\}$ is open in $X$ or equivalently if, for every closed $U \subset Y$, the set $\varphi_{+}^{-1}(U):=\{x \in X ; \varphi(x) \cap U \neq \emptyset\}$ is closed in $X$.

It is well known that, for every u.s.c. mapping $\varphi: X \multimap Y$, its graph $\Gamma_{\varphi}:=\{(x, y) \in X \times$ $Y ; y \in \varphi(x)\}$ is a closed subset of $X \times Y$. The reverse implication does not hold, in general.

On the other hand, if $\varphi: X \multimap Y$ is such that $\varphi(X) \subset K$, where $K \subset Y$ is a compact set and the graph $\Gamma_{\varphi}$ is closed, then $\varphi$ is u.s.c.

For u.s.c. maps $\varphi: X \multimap Y$ with compact values, if $K \subset X$ is compact, then so is $\varphi(K) \subset Y$.

The composition $\varphi_{2} \circ \varphi_{1}: X \multimap Z$ of two u.s.c. maps $\varphi_{1}: X \multimap Y$ and $\varphi_{2}: Y \multimap Z$ with compact values is again u.s.c. with compact values.

If a u.s.c. mapping $\varphi$ is single-valued, i.e. $\varphi: X \rightarrow Y$, then it is continuous.

For the proofs and more details as regards ANR-spaces and multivalued maps, we recommend $[7,8]$. 


\section{Approximable multivalued mappings}

In the entire text, all topological spaces are metric and all single-valued mappings are continuous. Moreover, we shall consider only upper semicontinuous (u.s.c.) multivalued mappings with compact values.

Let $X, Y$ be two metric spaces. We shall use the following notation: $f: X \rightarrow Y$, for singlevalued mappings, and $\varphi: X \multimap Y$, for multivalued mappings.

For a subset $A \subset X$ and $\varepsilon>0$, we let

$$
O_{\varepsilon}(A):=\{x \in X ; \exists y \in A \text { and } d(x, y)<\varepsilon\}
$$

where $d$ is the metric in $X$. For a singleton $x \in X$, i.e. for $A=\{x\}$, we simply put $O_{\varepsilon}(x)$.

Definition 3.1 (cf. [8-11]) Let $\varepsilon>0$ be a given real number. A map $f: X \rightarrow Y$ is an $\varepsilon$ approximation of $\varphi: X \multimap Y$ if $\Gamma_{f} \subset O_{\varepsilon}\left(\Gamma_{\varphi}\right)$, where

$$
\Gamma_{f}:=\{(x, y) \in X \times Y ; y=f(x)\} \quad \text { and } \quad \Gamma_{\varphi}:=\{(x, y) \in X \times Y ; y \in \varphi(x)\}
$$

are the graphs of $f$ and $\varphi$, respectively.

In the following, in $X \times Y$, we shall consider the max metric.

The following property is self-evident (cf. e.g. [8]):

$\Gamma_{\varphi} \subset O_{\varepsilon}\left(\Gamma_{\varphi}\right)$ if and only if, for every $x \in X$, there is $f(x) \in O_{\varepsilon}\left(\varphi\left(O_{\varepsilon}(x)\right)\right)$.

If $f: X \rightarrow Y$ is an $\varepsilon$-approximation of $\varphi: X \multimap Y$, then we write $f \in a(\varphi ; \varepsilon)$.

Now, we shall define the main, from our point of view, class of multivalued mappings.

Definition 3.2 A multivalued map $\varphi: X \multimap Y$ is called approximable (written $\varphi \in$ $A(X, Y))$ if the following two conditions are satisfied:

(a) for every $\varepsilon>0$, we have $a(\varphi ; \varepsilon) \neq \emptyset$,

(b) for every $\delta>0$, there exists $\varepsilon_{0}>0$ such that, for every $\varepsilon\left(0<\varepsilon \leq \varepsilon_{0}\right)$, if $f, g \in a(\varphi ; \varepsilon)$, then there exists a homotopy $h: X \times[0,1] \rightarrow Y$ linking $f$ and $g$ such that $h_{t} \in a(\varphi ; \delta)$, for every $t \in[0,1]$, where $h_{t}(x)=h(x, t)$.

Let $U$ be an open subset of $X$ and let $\partial U$ denote its boundary. We let

$$
A_{U}(X):=\{\varphi \in A(X, Y) ; \operatorname{Fix}(\varphi) \cap \partial U=\emptyset\}
$$

where $\operatorname{Fix}(\varphi):=\{x \in X ; x \in \varphi(x)\}$.

We shall employ the following lemma.

Lemma 3.3 Assume that $X$ is a compact space and $\varphi \in A_{U}(X)$. Then there exists $\varepsilon_{1}>0$ such that, for every $f_{\varepsilon} \in a(\varphi ; \varepsilon)$, with $0<\varepsilon \leq \varepsilon_{1}$, we have

$$
\operatorname{Fix}\left(f_{\varepsilon}\right):=\left\{x \in X ; f_{\varepsilon}(x)=x\right\} \cap \partial U=\emptyset .
$$

Proof Let, on the contrary, for every $\varepsilon>0$, there exist $f_{\varepsilon} \in a(\varphi ; \varepsilon)$ such that $\operatorname{Fix}\left(f_{\varepsilon}\right) \cap \partial U \neq \emptyset$. We put $\varepsilon_{n}=\frac{1}{n}, n=1,2, \ldots$, and $f_{n} \in a(\varphi ; \varepsilon)$. 
For every $n$, there exists $x_{n} \in \partial U \cap \operatorname{Fix}\left(f_{n}\right)$. Since $f_{n} \in a(\varphi ; \varepsilon)$, for every $n$, we get $\left(\tilde{x}_{n}, \tilde{y}_{n}\right) \in$ $\Gamma_{\varphi}$ such that

$$
d\left(x_{n}, \tilde{x}_{n}\right)<\frac{1}{n} \quad \text { and } \quad d\left(x_{n}, \tilde{y}_{n}\right)<\frac{1}{n}, \quad \text { for } n=1,2, \ldots
$$

But $\partial U$ is a compact set, so we can assume that $\lim _{n \rightarrow \infty} x_{n}=x \in \partial U$. Consequently, in view of (1), we obtain $\lim _{n \rightarrow \infty} \tilde{x}_{n}=x$, and $\lim _{n \rightarrow \infty} \tilde{y}_{n}=x$. Using the fact that $\varphi$ is u.s.c., we deduce that $(x, x) \in \Gamma_{\varphi}$ and $x \in \partial U$. It means that $x \in \operatorname{Fix}(\varphi) \cap \partial U$, which is a contradiction.

Now, we shall define the appropriate notion of homotopy in $A_{U}(X)$.

Definition 3.4 Two maps $\varphi, \psi \in A_{U}(X)$ are called homotopic (written $\varphi \sim \psi$ ) if there exists $\chi \in A(X \times[0,1], X)$ such that $\chi(x, 0)=\varphi(x)$ and $\chi(x, 1)=\psi(x)$, for every $x \in X$, and $x \notin \chi(x, t)$, for every $x \in \partial U$ and $t \in[0,1]$.

To indicate how large the class $A(X, Y)$ is, we let

$$
\begin{aligned}
J(X, Y):= & \left\{\varphi: X \multimap Y ; \varphi \text { is u.s.c., } \varphi(x) \text { is an } R_{\delta}\right. \text {-set, } \\
& \text { for every } x \in X, \text { and } Y \text { is an ANR-space }\} .
\end{aligned}
$$

We recall the following propositions (see e.g. [9-11]).

Proposition 3.5 (cf. [11], Corollary 3.4) If $\varphi: X \multimap Y$ can be decomposed as

$$
\varphi: X=Y_{0} \stackrel{\varphi_{1}}{\longrightarrow} Y_{1} \stackrel{\varphi_{2}}{\longrightarrow} \cdots \stackrel{\varphi_{n}}{\longrightarrow} Y_{n}=Y
$$

where $X=Y_{0}$ is a compact ANR-space and $\varphi_{i} \in J\left(Y_{i-1}, Y_{i}\right), i=1, \ldots, n$, then $\varphi$ is approximable, i.e. $\varphi \in A(X, Y)$. In particular, if $X$ is a compact $A N R$-space, then $J(X, Y) \subset A(X, Y)$.

Proposition 3.6 (cf. [11], Theorem 2.5) Let $X$ be a compact space and $Y, Z$ be arbitrary spaces. If $\varphi_{1}: X \multimap Y$ and $\varphi_{2}: Y \multimap Z$ are approximable, i.e. if $\varphi_{1} \in A(X, Y)$ and $\varphi_{2} \in A(Y, Z)$, then so is $\varphi_{2} \circ \varphi_{1}: X \multimap Z$, i.e. $\varphi_{2} \circ \varphi_{1} \in A(X, Z)$.

As a direct consequence of Proposition 3.5 and Proposition 3.6, we can give the following corollary which is quite appropriate for the class of multivalued Poincaré operators considered in the two concluding sections.

Corollary 3.7 In particular, if $\varphi \in J(X, Y)$, where $X$ is a compact ANR-space, then $f \circ \varphi \in$ $A(X, Z)$, for any single-valued map $f: Y \rightarrow Z$.

Open Problem 1 Is it true that acyclic mappings $(c f .[7,8])$ defined on compact ANRspaces are approximable? Let us note that by the acyclicity, we mean the one in the sense of the Čech homology theory with rational coefficients.

Let $X$ be a compact space and $B$ be a closed subset of $X$. Assume that $r: X \rightarrow B$ is a continuous mapping and $\varphi \in A(B, B)$. Then we put $\tilde{\varphi}=i \circ \varphi \circ r$, where $i: B \rightarrow X$ is the inclusion map. 
Proposition 3.8 Under the above assumption, we claim that, for each $\rho>0$, there exists $\varepsilon_{0}>0$ such that, for any $\varepsilon\left(0<\varepsilon<\varepsilon_{0}\right)$, iff $\in a(\varphi ; \varepsilon)$, then $i \circ f \circ r \in a(\tilde{\varphi} ; \rho)$.

The proof of Proposition 3.8 is quite analogous to the one in [10], Proposition 3.1.2 (cf. also [8], Proposition 22.3.2). From Proposition 3.8, we immediately have the following.

Corollary 3.9 If $\varphi \in A(B, B)$, then $\tilde{\varphi} \in A(X, X)$.

\section{Fixed point index for approximable mappings}

Let $X$ be a compact ANR and $U$ be an open subset of $X$. We shall define the fixed point index:

$$
\text { Ind: } A_{U}(X) \rightarrow \mathbb{Z}
$$

where $\mathbb{Z}$ is the set of integers.

Let $\varphi \in A_{U}(X)$. According to Lemma 3.3, we choose $\varepsilon_{1}>0$. Then we use Definition 3.2(b), for $\delta=\varepsilon_{1}$, and we get $\varepsilon_{0}>0$ such that Definition 3.2(b) holds true. Let $f_{\varepsilon} \in a(\varphi ; \varepsilon)$. Thus, in view of Lemma 3.3, we have $\operatorname{Fix}\left(f_{\varepsilon}\right) \cap \partial U=\emptyset$. So the fixed point index $\operatorname{ind}\left(f_{\varepsilon}, U\right)$ of $f_{\varepsilon}$ is well defined (cf. [12]).

We put

$$
\operatorname{Ind}(\varphi, U)=\operatorname{ind}\left(f_{\varepsilon}, U\right)
$$

Let us observe that Definition 3.2(b) and Lemma 3.3 guarantee that the definition (2) does not depend on the choice of $f_{\varepsilon} \in a(\varphi ; \varepsilon)$.

In particular, if $\varphi \in A(X, X)$, we can define the Lefschetz number $\lambda(\varphi)$ of $\varphi$ by putting

$$
\lambda(\varphi)=\lambda(f)
$$

where $f$ is chosen according to Definition 3.2(b) and $\lambda(f)$ is the ordinary Lefschetz number of $f(c f$. e.g. [12]).

Below there are collected the most important properties of the above fixed point index $(c f .[8,10,13])$.

\section{Proposition 4.1}

(i) (Existence) If $\operatorname{Ind}(\varphi, U) \neq 0$, then $\operatorname{Fix}(\varphi) \cap U \neq \emptyset$.

(ii) (Excision) If $\{x \in U$; $x \in \varphi(x)\} \subset V \subset U$, then $\operatorname{ind}(\varphi, V)=\operatorname{ind}(\varphi, U)$, where $V$ is an open subset of $X$.

(iii) (Additivity) Let $U_{1}, U_{2}$ be two open subsets of $X$ such that $U=U_{1} \cup U_{2}, U_{1} \cap U_{2}=\emptyset$ and $\operatorname{Fix}(\varphi) \cap\left(\bar{U} \backslash\left(U_{1} \cup U_{2}\right)\right)=\emptyset$, then

$$
\operatorname{Ind}(\varphi, U)=\operatorname{Ind}\left(\varphi, U_{1}\right)+\operatorname{Ind}\left(\varphi, U_{2}\right)
$$

(iv) (Homotopy) If $\varphi, \psi \in A_{U}(X)$ are homotopic, then $\operatorname{Ind}(\varphi, U)=\operatorname{Ind}(\psi, U)$.

(v) (Normalization) If $\varphi \in A(X, X)$, then $\lambda(\varphi)=\operatorname{Ind}(\varphi, X)$, where $\lambda(\varphi)$ stands for the ordinary Lefschetz number for $\varphi$. 
Observe that from the properties (i) and (v) in Proposition 4.1, we have the following.

Corollary 4.2 If $\varphi \in A(X, X)$ and $\lambda(\varphi) \neq 0$, then $\operatorname{Fix}(\varphi) \neq \emptyset$.

Let $B \subset X$ also be a compact ANR-space and $\varphi \in A_{U}(X)$ be such that $\varphi(X) \subset B$. Let $\varphi_{1}: B \multimap B$ be defined by the formula $\varphi_{1}(x)=\varphi(x)$, for every $x \in B$. Then $\varphi_{1} \in A_{U \cap B}(B)$ and $\operatorname{Ind}(\varphi, U)=\operatorname{Ind}\left(\varphi_{1}, U \cap B\right)$.

Let $X$ be a compact ANR-space and $r: X \rightarrow B$ be a retraction map. According to Corollary 3.9, if $\varphi \in A(B, B)$, then $\tilde{\varphi} \in A(X, X)$.

Observe that

$$
\operatorname{Fix}(\varphi)=\operatorname{Fix}(\tilde{\varphi}) \subset B
$$

Let $V$ be an open subset of $B$ such that $\operatorname{Fix}(\varphi) \cap \partial V=\emptyset$. We let $U=r^{-1}(V)$. Then $U$ is an open subset of $X$ such that $\partial U \cap \operatorname{Fix}(\tilde{\varphi})=\emptyset$. Consequently $\operatorname{Ind}(\varphi, V)$ and $\operatorname{Ind}(\tilde{\varphi}, U)$ are well defined. Using the excision property (ii) of the fixed point index, we get

$$
\operatorname{Ind}(\varphi, V)=\operatorname{Ind}(\tilde{\varphi}, U)
$$

\section{Essential fixed points}

In this section, we assume that all the spaces, under our consideration, are compact. Hence, let $(X, d)$ be a compact space. For a given point $x_{0} \in X$ and $\varepsilon>0$, we denote by

$$
O_{\varepsilon}\left(x_{0}\right):=\left\{x \in X ; d\left(x, x_{0}\right)<\varepsilon\right\} \text { and } \overline{O_{\varepsilon}\left(x_{0}\right)}:=\left\{x \in X ; d\left(x, x_{0}\right) \leq \varepsilon\right\}
$$

the open and closed balls in $X$, respectively.

Definition 5.1 Let $x_{0}$ be an isolated fixed point of $\varphi \in A(X, X)$. We say that $x_{0} \in X$ is an essential fixed point of $\varphi$ if, for every $\varepsilon>0$, there exists $\delta=\delta(\varepsilon)>0$ such that if $f \in a(\varphi ; \delta)$, then $\operatorname{Fix}(f) \cap O_{\varepsilon}\left(x_{0}\right) \neq \emptyset$.

Observe that if $\varphi$ is a single-valued mapping, then the essentiality in the sense of Definition 5.1 coincides with the one presented in [1], Definition 2.1. Concretely, an isolated fixed point $x_{0}$ of a single-valued mapping $f: X \rightarrow X$ is essential if, for every open $\varepsilon$ neighborhood $O_{\varepsilon}\left(x_{0}\right)$ of $x_{0}$, there exists $\delta=\delta(\varepsilon)>0$ such that any map $g: X \rightarrow X$ which is ' $\delta$-near' to $f$, i.e. $\sup _{x \in X} d(f(x), g(x))<\delta$, has a fixed point in $O_{\varepsilon}\left(x_{0}\right)$.

For a given $\varphi \in A(X, X)$, we put

$$
\operatorname{Ess}(\varphi):=\{x \in \operatorname{Fix}(\varphi) ; x \text { is an essential fixed point of } \varphi\}
$$

We have the following.

Theorem 5.2 Let $X$ be a compact ANR-space and $\varphi \in A(X, X)$. Assume further that $x_{0} \in$ Fix $(\varphi)$ is an isolated fixed point and $U$ be an open subset of $X$ such that $x_{0} \in U$ and $\operatorname{Fix}(\varphi) \cap$ $\partial U=\emptyset$. Then $\operatorname{Ind}(\varphi, U) \neq 0$ implies that $x_{0} \in \operatorname{Ess}(\varphi)$. 
Proof Letting $\varepsilon_{0}>0$, we can assume without any loss of generality that $O_{\varepsilon_{0}}\left(x_{0}\right) \subset U$, where $x_{0} \in \operatorname{Fix}(\varphi)$ is an isolated fixed point such that $x_{0} \in U$. From the excision property of the fixed point index, it then follows that $\operatorname{Ind}\left(\varphi, O_{\varepsilon_{0}}\left(x_{0}\right)\right)=\operatorname{Ind}(\varphi, U) \neq 0$. Applying Lemma 3.3 we can take $\varepsilon_{1}>0$ such that if $f_{\varepsilon} \in a(\varphi ; \varepsilon)$, for every $0<\varepsilon \leq \varepsilon_{1}$, then $\operatorname{Fix}\left(f_{\varepsilon}\right) \cap \partial O_{\varepsilon_{0}}\left(x_{0}\right)=\emptyset$. Thus, $\operatorname{ind}\left(f_{\varepsilon}, O_{\varepsilon_{0}}\left(x_{0}\right)\right)$ is well defined.

Now, for $\delta=\varepsilon_{1}$, we apply condition (b) from Definition 3.2, by which we obtain $\varepsilon_{2}>0$ such that, for every $0<\varepsilon \leq \varepsilon_{2}$, all the maps $f_{\varepsilon}, g_{\varepsilon} \in a(\varphi ; \varepsilon)$ are $\varepsilon_{1}$-homotopic, i.e. there exists a homotopy $h: X \times[0,1] \rightarrow X$, linking $f$ with $g$ such that $h_{t}: X \rightarrow X, h_{t}(x)=h(x, t)$ belongs to $a\left(\varphi ; \varepsilon_{1}\right)$.

We can assume that $\varepsilon_{2} \leq \varepsilon_{1}$. Consequently, for every two mappings $f_{\varepsilon}, g_{\varepsilon} \in a\left(\varphi ; \varepsilon_{2}\right)$, we have $\operatorname{ind}\left(f_{\varepsilon}, O_{\varepsilon_{1}}\left(x_{0}\right)\right)=\operatorname{ind}\left(g_{\varepsilon}, O_{\varepsilon_{1}}\left(x_{0}\right)\right)$, for every $0<\varepsilon \leq \varepsilon_{2}$.

Finally, it follows from the definition of the fixed point index for $\varphi$ that

$$
\operatorname{Ind}\left(\varphi, O_{\varepsilon_{0}}\left(x_{0}\right)\right)=\operatorname{ind}\left(f_{\varepsilon}, O_{\varepsilon_{0}}\left(x_{0}\right)\right) \neq 0
$$

for every $0<\varepsilon \leq \varepsilon_{2}$. Hence, for every $0<\varepsilon \leq \varepsilon_{2}$ and for all $f_{\varepsilon} \in a\left(\varphi ; \varepsilon_{2}\right)$, we can deduce that $\operatorname{Fix}\left(f_{\varepsilon}\right) \cap O_{\varepsilon_{0}}\left(x_{0}\right) \neq \emptyset$, which completes the proof.

Let $X$ be a compact ANR-space and $B$ be a retract of $X$. In view of the arguments presented in the foregoing section for $\varphi \in A(B, B)$, we denote by $\tilde{\varphi} \in A(X, X)$ the map defined by the formula $\tilde{\varphi}=i \circ \varphi \circ r$, where $r$ is a retraction map and $i$ is an inclusion.

The following proposition is obvious.

Proposition 5.3 If $x_{0} \in \operatorname{Ess}(\tilde{\varphi})$, then $x_{0} \in \operatorname{Ess}(\varphi)$.

The reverse implication is an open problem.

Let $X$ be a compact ANR-space. We let

$$
A^{0}(X, X):=\{\varphi \in A(X, X) ; \operatorname{dim} \operatorname{Fix}(\varphi)=0\}
$$

where $\operatorname{dim}(\cdot)$ stands for the topological (Lebesgue covering) dimension (see e.g. [14]).

Lemma 5.4 ( $c f .[14])$ Let $B$ be a compact space such that $\operatorname{dim} B=0$. Then, for every $x \in B$ and for every $\varepsilon>0$, there exists an open set $V \subset O_{\varepsilon}(x)$ such that $x \in V$ and $\partial V \cap B=\emptyset$.

Now, we are ready to give the main result of this paper.

Theorem 5.5 Let $X$ be a compact ANR-space and $\varphi \in A^{0}(X, X)$. If $\lambda(\varphi) \neq 0$, then $\operatorname{Ess}(\varphi) \neq$ $\emptyset$.

Proof By the hypothesis $\lambda(\varphi) \neq 0$, the fixed point set $\operatorname{Fix}(\varphi)$ is nonempty and compact, and $\operatorname{dim} \operatorname{Fix}(\varphi)=0$. Let $x_{0} \in \operatorname{Fix}(\varphi)$. In view of Lemma 5.4, there exists an open set $V \subset O_{\varepsilon}\left(x_{0}\right)$ such that $\partial V \cap \operatorname{Fix}(\varphi)=\emptyset$, for every $\varepsilon>0$.

Letting still $\Gamma=\{A \subset \operatorname{Fix}(\varphi)$; $A$ is nonempty and compact, and there exists a neighborhood $V$ of $A$ in $X$ such that $\partial V \cap \operatorname{Fix}(\varphi)=\emptyset$ and $\operatorname{ind}(\varphi, V) \neq 0\}$, observe that $\Gamma$ is nonempty, because $\operatorname{Fix}(t) \in \Gamma$. Thus, we can take $V=X$ and, by the normalization property of the 
fixed point index, we get

$$
\operatorname{Ind}(\varphi, V)=\lambda(\varphi) \neq 0 \text {. }
$$

We can consider in $\Gamma$ the partial ordering given by the inclusion of subsets of $X$.

Now, we shall verify the assumptions of the well-known Kuratowski-Zorn lemma. To do it, let us assume that $\left\{A_{i}\right\}_{i \in J}$ is the chain in $\Gamma$. We put $A_{0}=\bigcap\left\{A_{i} ; i \in J\right\}$.

To prove that $A_{0} \in \Gamma$, assume that $W$ is an open neighborhood of $A_{0}$ in $X$. We claim that there exists $i \in J$ such that $A_{i} \subset W$. Otherwise, if we would have assumed, on the contrary, that it is not so, then there is a family $B_{i}=(X \backslash W) \cap A_{i}, i \in J$, of nonempty, compact sets which has nonempty, compact intersection $B_{0}$. Therefore, $B_{0} \subset X \backslash W$, together with $B_{0} \subset$ $A_{0}$, which is a contradiction, and subsequently $A_{0} \in \Gamma$. Thus, in view of the KuratowskiZorn lemma, we get a minimal element $A_{*}$ in $\Gamma$.

We furthermore claim that $A_{*}$ is a singleton. Let $z \in A_{*}$. It is sufficient to show that $\{z\} \in$ $\Gamma$. Since $A_{*} \in \Gamma$, we obtain an open neighborhood $V_{*}$ of $A_{*}$ with the following properties: $V_{*} \subset V, \partial V_{*} \cap \operatorname{Fix}(\varphi)=\emptyset$ and $\operatorname{Ind}\left(\varphi, V_{*}\right) \neq 0$.

Let $W$ be an arbitrary open neighborhood of $z$ in $X$. Applying Lemma 5.4, we can choose an open neighborhood $V_{z}$ of $z$ in $V_{*} \cap W$ such that $\operatorname{Fix}(\varphi) \cap \partial V_{z}=\emptyset$. Since $A_{*}$ is a minimal element of $\Gamma$, the compact set $A_{*} \backslash V_{z}$ is not in $\Gamma$, and so there exists an open set $U \subset V_{*}$ such that $\left(A_{*} \backslash U_{z}\right) \subset U \subset V_{*}, \operatorname{Fix}(\varphi) \cap \partial U=\emptyset, \operatorname{Ind}(\varphi, U)=0$ and $\operatorname{Ind}\left(\varphi, V_{*}\right)=\operatorname{Ind}(\varphi, U \cup$ $\left.V_{z}\right)$.

Now, from the additivity property of the fixed point index, it follows that

$$
\operatorname{Ind}\left(\varphi, V_{*}\right)=\operatorname{Ind}\left(\varphi, V_{z}\right)+\operatorname{Ind}(\varphi, U) \neq 0,
$$

by which we arrive (in view of the above properties $\operatorname{Ind}\left(\varphi, V_{*}\right) \neq 0$ and $\operatorname{Ind}(\varphi, U)=0$ ) at $\operatorname{Ind}\left(\varphi, V_{z}\right) \neq 0$.

This already implies that $\{z\} \in \Gamma$ and, according to Theorem 5.2, we can conclude that $z$ is an essential fixed point of $\varphi$. This completes the proof.

Corollary 5.6 If $X$ is a compact $A R$-space and $\varphi \in A^{0}(X, X)$, then $\operatorname{Ess}(\varphi) \neq \emptyset$.

\section{Simple examples}

At first, we will give two simple illustrative examples of application of the main theorems.

Example 6.1 Consider the mapping $\varphi_{1}:[0,1] \multimap[0,1]$ defined as (see Figure 1 ):

$$
\varphi_{1}(x):= \begin{cases}{\left[0, \frac{1}{4}\right],} & \text { for } x \in\left[0, \frac{1}{4}\right], \\ \frac{1}{4}, & \text { for } x \in\left(\frac{1}{4}, \frac{1}{2}\right), \\ \left.\frac{1}{4}, \frac{3}{4}\right], & \text { for } x=\frac{1}{2}, \\ \frac{3}{4}, & \text { for } x \in\left(\frac{3}{2}, \frac{3}{4}\right), \\ 1, & \text { for } x=\frac{3}{4}, \\ 1, & \text { for } x \in\left(\frac{3}{4}, 1\right] .\end{cases}
$$

Since the graph $\Gamma_{\varphi_{1}}$ of $\varphi_{1}$ is closed and $[0,1]$ is a compact AR-space, $\varphi_{1}$ is obviously an upper semicontinuous map with convex, compact values, i.e. a special case of a $J$-mapping which is, according to Proposition 3.5, approximable. 


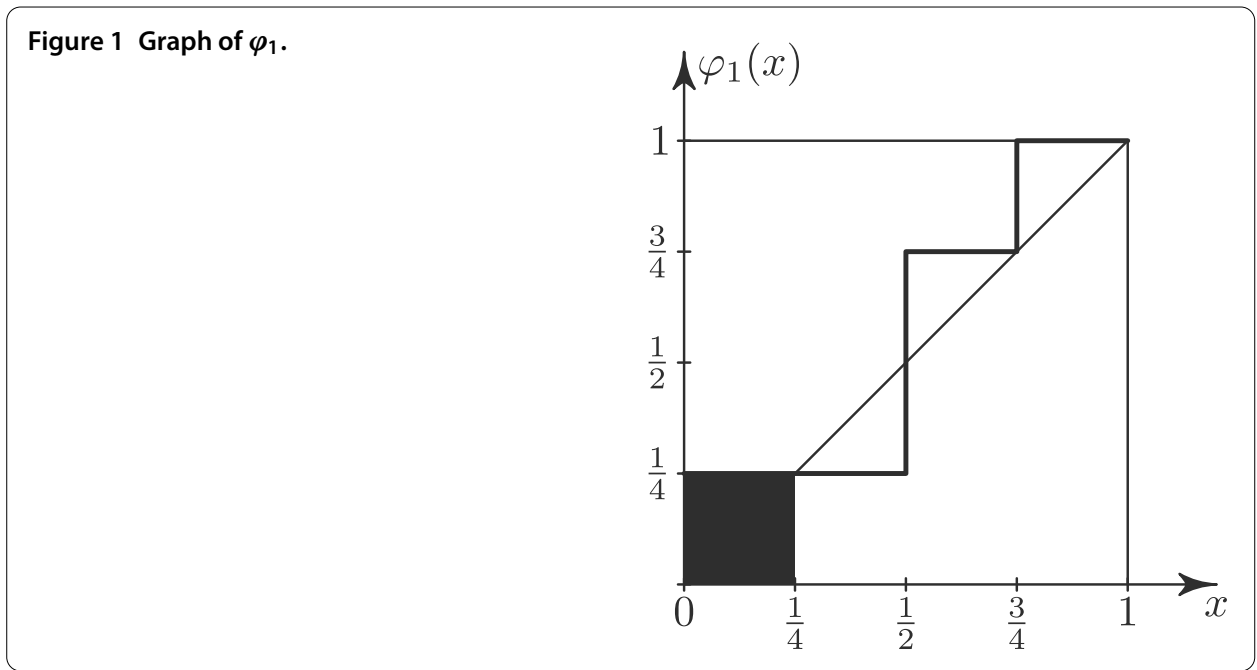

Hence, in order to apply Theorem 5.2 , let us observe that since the interval $\left[0, \frac{1}{4}\right]$ is a set of non-isolated fixed points such that $\operatorname{dim} \operatorname{Fix}\left(\varphi_{1}\right)=1$ (by which Theorem 5.5 cannot be applied here) and since the fixed point $\frac{3}{4}$ is, in view of $\operatorname{Ind}\left(\varphi_{1}, U_{\frac{3}{4}}\right)=0$, non-essential, we must concentrate on the fixed points $\frac{1}{2}$ and 1 . Since $\operatorname{Fix}\left(\varphi_{1}\right) \cap \partial U_{\frac{1}{2}}=\emptyset$ and $\operatorname{Ind}\left(\varphi_{1}, U_{\frac{1}{2}}\right) \neq 0$ as well as $\operatorname{Fix}\left(\varphi_{1}\right) \cap \partial U_{1}=\emptyset$ and $\operatorname{Ind}\left(\varphi_{1}, U_{1}\right) \neq 0$, both fixed points are, according to Theorem 5.2, essential.

Let us note that although the essentiality of 1 easily follows from the classical results for single-valued maps due to Fort, Jr. [3] and O'Neil [4], the appropriate application of Theorem 5.2 concerns the essential fixed point $\frac{1}{2}$.

Example 6.2 Consider the mapping $\varphi_{2}:[0,1] \multimap[0,1]$ defined as (see Figure 2):

$$
\varphi_{2}(x):= \begin{cases}{\left[0, \frac{1}{4}\right],} & \text { for } x=0, \frac{1}{4}, \\ 0, & \text { for } x \in\left(0, \frac{1}{4}\right), \\ \frac{1}{4}, & \text { for } x \in\left(\frac{1}{4}, \frac{1}{2}\right), \\ {\left[\frac{1}{4}, \frac{1}{2}\right],} & \text { for } x=\frac{1}{2}, \\ \frac{1}{2}, & \text { for } x \in\left(\frac{1}{2}, \frac{3}{4}\right), \\ {\left[\frac{1}{2}, \frac{3}{4}\right],} & \text { for } x=\frac{3}{4}, \\ \frac{3}{4}, & \text { for } x \in\left(\frac{3}{4}, 1\right), \\ {\left[\frac{3}{4}, 1\right],} & \text { for } x=1 .\end{cases}
$$

By the same reasoning as in Example 6.1, $\varphi_{2}$ is obviously an approximable $J$-mapping. Hence, in order to apply Theorem 5.2, resp. Corollary 5.6, it is enough to realize that $\lambda\left(\varphi_{2}\right)=1$ and $\operatorname{dim} \operatorname{Fix}\left(\varphi_{2}\right)=0$. Thus, $\operatorname{Ess}\left(\varphi_{2}\right) \neq \emptyset$.

Since $\operatorname{Ind}\left(\varphi_{2}, U_{\frac{1}{4}}\right)=\operatorname{Ind}\left(\varphi_{2}, U_{\frac{1}{2}}\right)=\operatorname{Ind}\left(\varphi_{2}, U_{\frac{3}{4}}\right)=\operatorname{Ind}\left(\varphi_{2}, U_{1}\right)=0$, there is (in view of Theorem 5.2) the only essential fixed point 0 . Let us note that, because of a multivalued character of $\varphi_{2}$, the essentiality of 0 cannot be this time deduced by the local application of classical results due to Fort, Jr. [3] and O'Neil [4]. On the other hand, the same easily follows from the locally applied Theorem 5.2. 


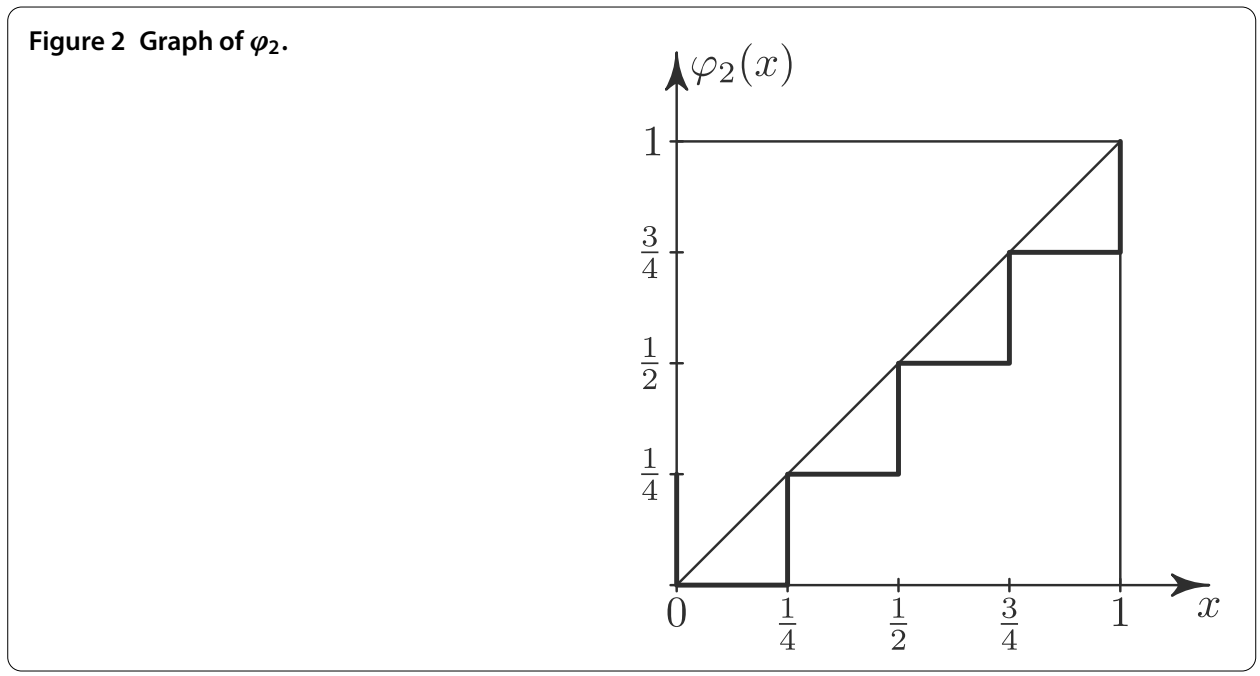

Now, we would like to discuss a possible application of Theorem 5.2 and Theorem 5.5 to scalar differential equations and inclusions. Hence, consider the scalar differential inclusion

$$
x^{\prime} \in F(t, x)
$$

where $F(t, x) \equiv F(t+\omega, x)$, for some $\omega>0$, and assume that $F:[0, \omega] \times \mathbb{R} \multimap \mathbb{R}$ is an upper semicontinuous mapping with convex, compact values, i.e. $F$ to be a Marchaud mapping. Let, furthermore, (6) be dissipative in the sense of Levinson, i.e.

$$
\exists D>0 \text { : } \limsup _{t \rightarrow \infty}|x(t)|<D \text {, for all solutions } x(\cdot) \text { of }(6) .
$$

This already implies ( $c f$. [15], pp.63-64) that, under $F(t, x) \equiv F(t+\omega, x)$, (6) is uniformly dissipative, i.e.

$$
\forall D_{0}>0 \exists \Delta t>0, D>0:\left(t_{0} \in \mathbb{R},\left|x_{0}\right|<D_{0}, t \geq t_{0}+\Delta t\right) \Rightarrow|x(t)|<D,
$$

for all solutions $x(\cdot)=x\left(\cdot ; t_{0}, x_{0}\right)$, satisfying $x\left(t_{0} ; t_{0}, x_{0}\right)=x_{0}$.

Defining the Poincaré translation operator along the trajectories of (6), $T_{\omega}: \mathbb{R} \multimap \mathbb{R}$, namely

$$
T_{\omega}\left(x_{0}\right):=\left\{x\left(\omega ; 0, x_{0}\right) ; x\left(\cdot ; 0, x_{0}\right) \text { is a solution of }(6) \text { with } x\left(0 ; 0, x_{0}\right)=x_{0}\right\},
$$

it is well known (cf. e.g. [7], Chapter 3.4) that, unlike in higher dimensions, $T_{\omega}^{n} \in J(\mathbb{R}, \mathbb{R})$, where

$$
T_{\omega}^{n}=\underbrace{T_{\omega} \circ \cdots \circ T_{\omega}}_{n \text {-times }}=T_{n \omega}
$$

for all $n \in \mathbb{N}$. Furthermore, each fixed point, say $\bar{x}_{0} \in T_{\omega}^{n}\left(\bar{x}_{0}\right)$, determines an $n \omega$-periodic solution $x(\cdot)=x\left(\cdot ; 0, \bar{x}_{0}\right)$ of $(6)$, because it can be entirely prolongated in an $\omega$-periodic way. 
On the other hand, although $n \omega$ need not be its minimal period, we have proved in [16] that if $n>1$ is minimal then, for each $m \in \mathbb{N}$, there exists a fixed point $\bar{x}_{m} \in T_{\omega}^{m}\left(\bar{x}_{m}\right)$ of $T_{\omega}^{m}$, determining a subharmonic $m \omega$-periodic solution of (6) with a minimal period. For $n=1$, the existence of a fixed point $\bar{x}_{1} \in T_{\omega}^{n}\left(\bar{x}_{1}\right)$, determining a harmonic $\omega$-periodic solution of (6), follows already from the generalised Levinson transformation theory (see [17] and the references therein).

In our context, condition (7) and subsequently (8) imply the existence of a sufficiently large $n_{0} \in \mathbb{N}$ such that, for every $n \geq n_{0},\left.T_{\omega}^{n}\right|_{[-D, D]} \in J([-D, D]$, $[-D, D])$. Thus, a fixed point of $\left.T_{\omega}^{n}\right|_{[-D, D]}:[-D, D] \multimap[-D, D]$ determines an $n \omega$-periodic solution for $(6)$, but in order this fixed point to be essential, we need to satisfy the assumptions of Theorem 5.2 or Corollary 5.6. In the latter case, it would mean to suppose still that $\operatorname{dim} \operatorname{Fix}\left(\left.T_{\omega}^{n}\right|_{[-D, D]}\right)=$ $\operatorname{dim} \operatorname{Fix}\left(\left.T_{n \omega}\right|_{[-D, D]}\right)=0$, which seems to be rather difficult to verify in general, because generically $\operatorname{dim} \operatorname{Fix}\left(\left.T_{\omega}^{n}\right|_{[-D, D]}\right)=1$ (cf. e.g. $\left.[18,19]\right)$, for multivalued maps $T_{\omega}$ in $\mathbb{R}$, i.e. in the lack of uniquely solvable Cauchy (initial value) problems for (6). If additionally

$$
F(t, x) \operatorname{sgn} x<0, \quad \text { for }|x| \geq D,
$$

then the interval $[-D, D]$ is obviously positively invariant under $\left.T_{\omega}\right|_{[-D, D]}$, by which only $\operatorname{dim} \operatorname{Fix}\left(\left.T_{\omega}\right|_{[-D, D]}\right)=0$ should be verified, but the same obstruction remains there again.

In the trivial case of uniqueness, $T_{\omega}: \mathbb{R} \rightarrow \mathbb{R}$ must be strictly increasing (otherwise, we get a contradiction) by which no purely subharmonic (i.e. those with $n>1) n \omega$-periodic solutions can exist. Moreover, this behavior highly increases the chance that

$$
\operatorname{dim} \operatorname{Fix}\left(\left.T_{\omega}^{n}\right|_{[-D, D]}\right)=\operatorname{dim} \operatorname{Fix}\left(\left.T_{\omega}\right|_{[-D, D]}\right)=0
$$

holds, even without (9).

Let us therefore give the last simple illustrative related example.

Example 6.3 Consider the scalar differential inclusion

$$
x^{\prime}+c x \in-F_{0}(x)+\cos t, \quad \text { with } c>1,
$$

where

$$
F_{0}(x):= \begin{cases}\arctan x, & \text { for }|x|<1 \\ \frac{\pi}{4}+\left[-\frac{\pi}{4}, 0\right], & \text { for } x=1 \\ -\frac{\pi}{4}+\left[0, \frac{\pi}{4}\right], & \text { for } x=-1 \\ 0, & \text { for }|x|>1\end{cases}
$$

Since condition (9) can easily be verified for $F(t, x):=-F_{0}(x)-c x+\cos t$, on $|x| \geq D$, where $D \in\left(\frac{1}{c}, 1\right)$, and $F(t, x) \equiv F(t+2 \pi, x), F(t, x) \equiv-F(t+\pi,-x)$, the associated Poincaré translation operator along the trajectories of $(10), T_{2 \pi}: \mathbb{R} \multimap \mathbb{R}$, satisfies $\left.T_{2 \pi}\right|_{[-1,1]}$ : $[-1,1] \multimap(-1,1)$. Observe that $\left.T_{2 \pi}\right|_{(-1,1)}$ is even single-valued, because $\arctan x$ is, for $|x| \leq 1$, Lipschitzian with constant $L=1$ as well as $c x$ with constant $c>1$.

Moreover, one can easily check that the differential equation

$$
x^{\prime}+c x=-\arctan x+\cos t
$$


has a unique $2 \pi$-periodic solution, provided only $c>1$; for more details, see e.g. [7], pp.354-355.

Because of an evident one-to-one correspondence between the fixed points $\bar{x}_{0}=$ $\left.T_{2 \pi}\right|_{(-1,1)}\left(\bar{x}_{0}\right)=\left.T_{2 \pi}\right|_{[-1,1]}\left(\bar{x}_{0}\right)$ of $\left.T_{2 \pi}\right|_{[-1,1]}$ and $2 \pi$-periodic solutions $x\left(\cdot ; 0, \bar{x}_{0}\right) \equiv x(\cdot+2 \pi$; $\left.0, \bar{x}_{0}\right)$ of (11) as well as (10), the unique fixed point $\bar{x}_{0}$ must be, in view of $\operatorname{dim} \operatorname{Fix}\left(\left.T_{2 \pi}\right|_{[-1,1]}\right)=$ 0 , essential by means of Corollary 5.6. The determined $2 \pi$-periodic solution $x\left(\cdot ; 0, \bar{x}_{0}\right)$ of (10) can be therefore called discretely essential.

Remark 6.4 Since $F(t, x) \equiv-F(t+\pi,-x)$, we can still prove by means of the modified operator $\left.\tilde{T}_{\pi}\right|_{[-1,1]}=-\left.T_{\pi}\right|_{[-1,1]}:[-1,1] \multimap(-1,1)$ that the unique discretely essential $2 \pi$ periodic solution $x\left(\cdot ; 0, \bar{x}_{0}\right) \equiv x\left(\cdot+2 \pi ; 0, \bar{x}_{0}\right)$ of $(10)$ in Example 6.3 is $\pi$-antiperiodic, i.e. $x\left(\cdot ; 0, \bar{x}_{0}\right) \equiv-x\left(\cdot+\pi ; 0, \bar{x}_{0}\right)$.

\section{Application and concluding remarks}

In higher dimensions, i.e. in $\mathbb{R}^{n}$ with $n>1$, the principle of essential fixed points of the Poincaré translation operators $\left.T_{\omega}\right|_{\overline{O_{D}(0)}}: \overline{O_{D}(0)} \multimap \overline{O_{D}(0)}$, resp. their iterates $\left.T_{\omega}^{k}\right|_{\overline{O_{D}(0)}}: \overline{O_{D}(0)} \multimap \overline{O_{D}(0)}$, along the trajectories of $\omega$-periodic dissipative systems, is quite analogous (see Corollary 3.7). In fact, it again consists in verification of the property

$$
\operatorname{dim} \operatorname{Fix}\left(T_{\omega}^{k} \mid \overline{O_{D}(0)}\right)=\operatorname{dim} \operatorname{Fix}\left(T_{k \omega} \mid \overline{O_{D}(0)}\right)=0 .
$$

However, since this verification is not an easy task in general, we have not formulated so far the related statement as a theorem.

Nevertheless, we can finally state as a theorem at least a particular case of it.

Theorem 7.1 Let $x^{\prime} \in F(t, x)$ be an $\omega$-periodic dissipative Marchaud system, i.e. let $F:[0, \omega] \times \mathbb{R}^{n} \multimap \mathbb{R}^{n}$ be an upper semicontinuous map with convex, compact values and $F(t, x) \equiv F(t+\omega, x)$ such that (7) is satisfied for all solutions of (6). If inclusion (6) possesses at most a finite number of subharmonic solutions $x(\cdot)$, i.e. those with $x(t) \equiv x(t+k \omega), k \in \mathbb{N}$, then at least one of them exists to be discretely essential. In other words, then for some $k_{0} \in \mathbb{N}$ there exists an essential fixed point $\bar{x}_{0} \in O_{D}(0) \subset \mathbb{R}^{n}$ of the associated Poincaré operator $T_{k_{0} \omega}=T_{\omega}^{k_{0}}: \overline{O_{D}(0)} \multimap O_{D}(0)$, determining this discretely essential subharmonic.

Corollary 7.2 If a periodic dissipative system of Marchaud inclusions possesses at most a finite number of entirely bounded solutions, then it admits a discretely essential (subharmonic) periodic solution.

Open Problem 2 Is, under the same assumptions, the conclusion of Theorem 7.1, resp. Corollary 7.2, true for $k_{0}=1$, i.e. is among the existing ( $c f$. [17]) harmonic solutions at least one to be discretely essential?

Remark 7.3 For the same conclusion of Theorem 7.1, resp. Corollary 7.2, the Marchaud maps $F$ can be replaced by more general upper Carathéodory maps with convex, compact values. For their definition and more details, see e.g. $[7,8]$

Remark 7.4 It can be proved that the discretely essential solution of (10), in Example 6.3, is at the same time essential as a fixed point of the Hammerstein-type operator 
$\int_{0}^{2 \pi} G(t, s)\left[-F_{0}(x(t))+\cos t\right] d t$, where

$$
G(t, s):= \begin{cases}\frac{e^{-c}(t-s+2 \pi)}{1-e^{-2 \pi c}}, & \text { for } 0 \leq t \leq s \leq 2 \pi, \\ \frac{e^{-c}(t-s)}{1-e^{-2 \pi c}}, & \text { for } 0 \leq s \leq t \leq 2 \pi .\end{cases}
$$

\section{Competing interests}

The authors declare that they have no competing interests.

\section{Authors' contributions}

All authors contributed equally in this article. They read and approved the final manuscript.

\section{Author details}

'Department of Mathematical Analysis and Applications of Mathematics, Faculty of Science, Palacký University, 17. listopadu 12, Olomouc, 771 46, Czech Republic. ${ }^{2}$ Institute of Mathematics, University of Kazimierz Wielki, Weyssenhoffa 11, Bydgoszcz, 85-072, Poland.

\section{Acknowledgements}

The first author was supported by the grant No. 14-06958S 'Singularities and impulses in boundary value problems for nonlinear ordinary differential equations' of the Grant Agency of the Czech Republic.

\section{Received: 25 January 2016 Accepted: 28 June 2016 Published online: 15 July 2016}

\section{References}

1. Andres, J, Górniewicz, L: On essential fixed points of compact mappings on arbitrary absolute neighborhood retracts and their application to multivalued fractals. Int. J. Bifurc. Chaos 26(3), 1650041 (2016)

2. Andres, J, Górniewicz, L: Recent results on the topological fixed point theory of multivalued mappings: a survey. Fixed Point Theory Appl. 2015, Article ID 184 (2015). doi:10.1186/s13663-015-0432-0

3. Fort, MK Jr.: Essential and non essential fixed points. Am. J. Math. 72, 315-322 (1950)

4. O'Neil, B: Essential sets and fixed points. Am. J. Math. 75, 497-509 (1953)

5. Del Prete, I, Naimpally, S, di lorio, M: Essential fixed points of functions and multifunctions. Real Anal. Exch. 25(1), 369-382 (1999)

6. Jiang, J: Essential fixed points of the multivalued mappings. Sci. China Math. 11(3), 293-298 (1962)

7. Andres, J, Górniewicz, L: Topological Fixed Point Principles for Boundary Value Problems. Kluwer Academic, Dordrecht (2003)

8. Górniewicz, L: Topological Fixed Point Theory of Multivalued Mappings, 2nd edn. Springer, Berlin (2006)

9. Ben-El-Mechaiekh, H: Spaces and maps approximation and fixed points. J. Comput. Appl. Math. 113(1-2), 283-308 (2000)

10. Górniewicz, L, Granas, A, Kryszewski, W: On the homotopy method in the fixed point index theory of multivalued mappings of compact absolute neighborhood retracts. J. Math. Anal. Appl. 161(2), 457-473 (1991)

11. Górniewicz, L, Lassonde, M: Approximation and fixed points for compositions of $R_{\delta}$-maps. Topol. Appl. 55(3), 239-250 (1994)

12. Granas, A, Dugundji, J: Fixed Point Theory. Springer, Berlin (2003)

13. Bader, R, Kryszewski, W: Fixed-point index for compositions of set-valued maps with proximally $\infty$-connected values on arbitrary ANR's. Set-Valued Anal. 2(3), 459-480 (1994)

14. Engelking, R: Dimension Theory. North-Holland, Amsterdam (1978)

15. Cheban, DN: Global Attractors of Set Valued Dynamical and Control Systems. Nova Science Publishers, New York (2010)

16. Andres, J, Fürst, T, Pastor, K: Period two implies all periods for a class of ODEs: a multivalued map approach. Proc. Am. Math. Soc. 135(10), 3187-3191 (2007)

17. Andres, J, Górniewicz, L: Periodic solutions of dissipative systems revisited. Fixed Point Theory Appl. 2006, Article ID 65195 (2006)

18. Augustynowicz, A, Dzedzej, Z, Gel'man, BD: The solution set to BVP for some functional differential inclusions. Set-Valued Anal. 6(3), 257-263 (2002)

19. Bader, R, Gel'man, BD, Kamenskii, M, Obukhovskii, V: On the topological dimension of the solutions sets for some classes of operator and differential inclusions. Discuss. Math., Differ. Incl. Control Optim. 22(1), 17-37 (2002) 